\title{
2018 Electronics Travel Awards for Postdoctoral Fellows and PhD Students Announcement
}

\author{
Mostafa Bassiouni ${ }^{1, *}$ and Electronics Editorial Office ${ }^{2}$ \\ 1 Department of Computer Science, University of Central Florida, Orlando, FL 32816, USA \\ 2 Room 803, Building No. 3, Zijin Digital Park, Haidian District, Beijing 100190, China; electronics@mdpi.com \\ * Correspondence: bassi@cs.ucf.edu
}

Received: 5 March 2018; Accepted: 6 March 2018; Published: 7 March 2018

Electronics launched, in 2018, the Travel Awards for Postdoctoral Fellows and PhD Students for the first time. We received a large number of highly meritorious applications from postdoctoral researchers and $\mathrm{PhD}$ students from all over the world. It was not an easy task to select the top candidates. Nevertheless, with the assistance of Electronics Editorial Office, we identified outstanding candidates for Postdoctoral fellows and PhD students, respectively. As Editor-in-Chief of Electronics, I am pleased to announce the following winners of the 2018 Electronics Travel Awards: Mr. Spyridon-Nektarios Daskalakis for the PhD Students Travel Award, and Dr. Yao-Feng Chang for the Postdoctoral Fellows Travel Award.

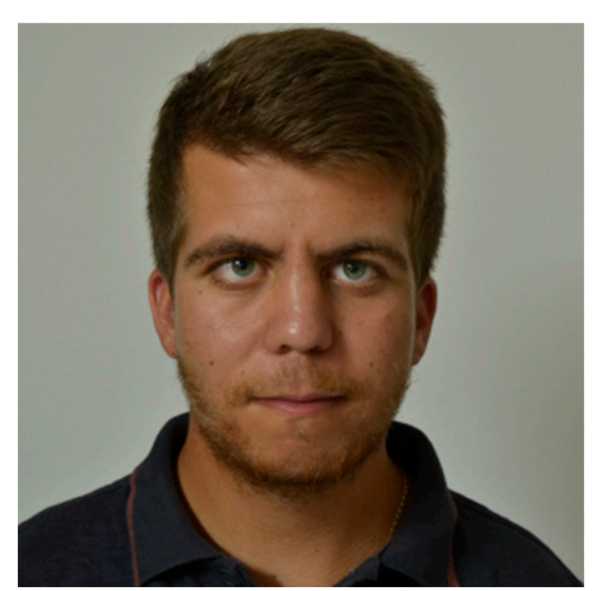

(a)

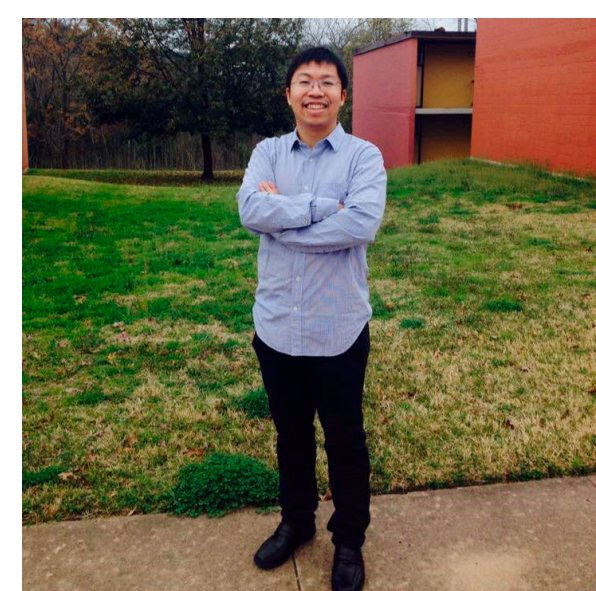

(b)

Figure 1. The winners of 2018 Electronics Travel Awards; (a) Mr. Spyridon-Nektarios Daskalakis; (b) Dr. Yao-Feng Chang.

Mr. Spyridon-Nektarios Daskalakis, shown in Figure 1a, was born in Heraklion, Greece, in 1991. He received with excellence his Engineering Diploma and the MSc in Electronic and Computer Engineering from the Technical University of Crete (TUC) in 2014 and 2016, respectively. He is currently working toward the PhD degree at the School of Engineering \& Physical Science of Heriot Watt University, Edinburgh UK. His current research interests include low-cost wireless sensor networks and RF energy harvesting. In particular, he focuses on backscatter radio communication, batteryless sensors, PCB design, low-cost software-defined radio, environmental sensing and RF energy harvesting. He has received a fellowship award for his project Aristeos (olive fly detection and monitoring with wireless sensor network) from the Clinton Global Initiative University 2014, Phoenix, Arizona USA, and the Onassis Foundation graduate studies 2015/16 scholarship. He is a co-founder of the kaloudia.com platform. Finally, he was the recipient of two short-term scientific mission grants from COST Action 
IC1301 WiPE in Electrical and Computer Engineering, Georgia Institute of Technology (2016), and the Centre Tecnològic de Telecomunicacions de Catalunya (2015). He has been a member of the IEEE since 2012, a member of the the IEEE Microwave Theory and Techniques Society, the IEEE Council on RFID and the IEEE Sensors Council. In the first year of his $\mathrm{PhD}$, he already has 2 journal publications (MTT-S) and 5 international conference publications (IEEE MTT-S IMS, IEEE MTT-S IMWS, IEEE Sensors, EuMW, ACES). Finally, he was recently awarded one of the Heriot-Watt University School of Engineering and Physical Sciences 1st year Postgraduate Research Prizes. He will use the Electronics Travel Award to attend the International Microwave Symposium (IMS2018) in Philadelphia, USA, 10-15 June 2018.

Dr. Yao-Feng Chang, shown in Figure 1b, is a R\&D Emerging Memory Device Engineer at Micron Technology, Inc. He received a BS degree in Electrical Engineering from National Sun Yat-sen University in 2007; the MS degree in Electronics Engineering from National Chiao Tung University in 2009; and the $\mathrm{PhD}$ degree from the Department of Electrical and Computer Engineering at The University of Texas at Austin in 2015. From 2011 to 2012, he was an intern at the SEMATECH, Austin, in the ReRAM Program. From 2013 to 2014, he was a research scientist at PrivaTran (a build-up company). He has published over 40 journal publications and 30 conference proceedings and has been awarded numerous research awards. He received the Senior Prize of the Ben Streetman Awards from the J. J. Pickle Research Center for his work on studies of resistive switching memories. His research since 2009 has focused on three topics: (1) $\mathrm{SiO}_{\mathrm{x}}$ - and $\mathrm{SiN}_{\mathrm{x}}$-based ReRAM fabrication, characterization, and mechanism understanding. (2) Stateful Boolean logic and synaptic Post-Moore computing, and bio-inspiration biomimetic systems in electronic devices. (3) Selector integration for large-scale low-power array design. He will use the Electronics Travel Award to attend the 233rd ECS Meeting held in Seattle, WA, USA, 13-17 May 2018.

The editors, managing editor and editorial board members join me in congratulating Mr. Spyridon-Nektarios Daskalakis and Dr. Yao-Feng Chang on winning the 2018 Electronics Travel Awards. Electronics is proud to support these young researchers working in the field of electronics and wish them further success in their careers. We are grateful to all who submitted applications-thank you for letting us get to know you and your work. We hope all of you continue to pay attention to Electronics in the future. We will continue to offer the Electronics Travel Awards in future years to support young researchers who work within the scope of Electronics. Finally, we are grateful to MDPI for their generous support of young scholars, helping them to present their work in impactful international conferences.

(C) 2018 by the authors. Licensee MDPI, Basel, Switzerland. This article is an open access article distributed under the terms and conditions of the Creative Commons Attribution (CC BY) license (http:/ / creativecommons.org/licenses/by/4.0/). 